\title{
The Near Subnormal Weighted Shift and Recursiveness
}

\author{
R. Ben Taher and M. Rachidi \\ Group of DEFA, Department of Mathematics and Informatics, Faculté des Sciences, Université Moulay Ismail, \\ BP 11201, Zitoune, Méknés, Morocco \\ Correspondence should be addressed to R. Ben Taher; bentaher89@hotmail.fr
}

Received 19 October 2012; Accepted 14 February 2013

Academic Editor: Chuanxi Qian

Copyright ( 2013 R. Ben Taher and M. Rachidi. This is an open access article distributed under the Creative Commons Attribution License, which permits unrestricted use, distribution, and reproduction in any medium, provided the original work is properly cited.

We aim at studying the near subnormality of the unilateral weighted shifts, whose moment sequences are defined by linear recursive relations of finite order. Using the basic properties of recursive sequences, we provide a natural necessary condition, that ensure the near subnormality of this important class of weighted shifs. Some related new results are established; moreover, applications and consequences are presented; notably the notion of near subnormal completion weighted shift is implanted and explored.

\section{Introduction}

Let $\alpha=\left\{\alpha_{n}\right\}_{n \in \mathbb{N}}$ be a bounded sequence of $\mathbb{R}($ or $\mathbb{C}$ ) and $\mathscr{H}$ a separable Hilbert space of basis $\left\{e_{n}\right\}_{n \geq 0}$. The unilateral weighted shift with weight sequence $\alpha$ is defined by $W_{\alpha} e_{n}=$ $\alpha_{n} e_{n+1}$. The moments of the operator $W_{\alpha}$ are given by

$$
\gamma_{0}=1, \quad \gamma_{k} \equiv \gamma_{k}(\alpha):=\alpha_{0}^{2} \alpha_{1}^{2} \cdots \alpha_{k-1}^{2} \quad \text { if } k>0 .
$$

It is well known that the bounded operator $W_{\alpha}$ can never be normal, and it is hyponormal if and only if $\left|\alpha_{n}\right| \leq\left|\alpha_{n+1}\right|$ (see [1-3]). For a given positive $D \in B(\mathscr{H})$, the space of bounded operators on $\mathscr{H}$, an operator $T \in B(\mathscr{H})$, is called a $D$-near subnormal operator if there exists a constant $m>0$ satisfying $m D \geq T^{*} D T$. An hyponormal operator $T \in B(\mathscr{H})$ is called near subnormal if it is $Q_{T}$-near subnormal, where $Q_{T}=T^{*} T-T T^{*}\left(Q_{T} \geq 0\right)$. Some necessary and sufficient conditions guaranteeing the near subnormality for unilateral weighted shifts have been established in $[4,5]$.

In this paper, we are interested in studying the near subnormal unilateral weighted shifts, when the sequence $\gamma \equiv$ $\left\{\gamma_{n}\right\}_{n \in \mathbb{N}}$ of moments $\alpha$ satisfies the following linear recursive relation of order $r \geq 2$ :

$$
\gamma_{n+1}=a_{0} \gamma_{n}+a_{1} \gamma_{n-1}+\cdots+a_{r-1} \gamma_{n-r+1} \quad \text { for every } n \geq r,
$$

where $\gamma_{0}, \gamma_{1}, \ldots, \gamma_{r-1}$ are the initial data and $a_{0}, a_{1}, \ldots, a_{r-1}$ are fixed real or complex numbers with $a_{r-1} \neq 0$. Such sequences are widely studied in the literature, generally called $r$-generalized Fibonacci sequences (see [6] and references therein). A weighted shift $W_{\alpha}$ such that $\gamma=\left\{\gamma_{n}\right\}_{n \geq 0}$ is a sequence (2) is called a recursive weighted shift. Our motivation in considering sequence (2) is inspired from the fact that every weighted shift is norm-limit of recursively generated weighted shifts (for further information we refer, to $[1,2,7]$, e.g.). . On the other hand, these sequences play a central role in the characterization of the subnormality via the truncated moment problem (see $[1,2,7,8]$ ). It turns out that following Curto-Fialkow's approach, the roots of the polynomial $P(z)=z^{r}-a_{0} z^{r-1}-\cdots-a_{r-1} z-$ $a_{r}=\prod_{i=1}^{s}\left(z-\lambda_{i}\right)^{k_{i}}$, called the characteristic polynomial of (2), play an important role, for establishing properties of subnormality, via Berger's Theorem. Moreover, in the process of construction of the generating measure, related to the truncated moment problem and subnormality of $[8,9]$, it reveals some significant obstructions when all roots $\lambda_{j}(1 \leq$ $j \leq s$ ) are not simple; some additional conditions on the initial data $\gamma_{0}, \gamma_{1}, \ldots, \gamma_{r-1}$ are necessary for one thing, to guarantee the existence of the generating measure. On the other hand, it was established in [10] that every $\gamma_{n}$ can be expressed as a moment of distribution of discrete support.

In this paper, we describe a deductive reasoning to prove that appending a mild hypotheses to the natural necessary condition for the existence of the hyponormal operators is sufficient, so that the unilateral weighted shifts, whose associate moment sequences satisfy (1), are near subnormal (Section 2). The main tool employed is the Binet formula of 
sequence (2) (see [6]). We then uprise to the near subnormal completion problem (NSCP) of order $m$, while at the same time evolving the subnormal completion problem (see [2]), the case $m=2$ is examined and solved. In the last section, we are interested in stretching our study for characterizing the near subnormality of a recursive weighted shift $W_{\alpha}$ such that the moment sequence $\gamma=\left\{\gamma_{n}\right\}_{n \geq 0}$ satisfies (2); we employ some results on the moment of distributions of discrete support (see $[8,10])$. The construction of the representing distribution is derived from the Binet formula of sequence (2); we preclude to set any condition on the initial data $\gamma_{0}, \gamma_{1}, \ldots, \gamma_{r-1}$. The closed relation of the near subnormality and the subnormality of weighted shifts is discussed.

\section{Recursive Sequences and Near Subnormality of Unilateral Weighted Shifts}

Let $T \in B(\mathscr{H})$ be a hyponormal operator and a sequence $\alpha=$ $\left\{\alpha_{n}\right\}_{n \in \mathbb{N}}$ such that $T e_{n}=\alpha_{n} e_{n+1}$, for all $n \geq 1$. It was established in [4] that if $0<\alpha_{1}<\alpha_{2}<\alpha_{3}<\cdots$, then $T$ is near subnormal if and only if $\sup _{n \geq 1}\left\{\alpha_{n}\left(\left(\alpha_{n+1}^{2}-\alpha_{n}^{2}\right) /\left(\alpha_{n}^{2}-\alpha_{n-1}^{2}\right)\right)^{1 / 2}\right\}<+\infty$, where $\alpha_{0}=0$. This characterization of near subnormality for unilateral weighted shifts is more practical and adequate in this section. Suppose that the moment sequence $\gamma=\left\{\gamma_{n}\right\}_{n \in \mathbb{N}}$ of the weighted shift $\alpha$ satisfies (2). Expression (1) shows that $\alpha_{n}^{2}=\gamma_{n+1} / \gamma_{n}$, for $n \geq 1$; as a matter of fact, we formulate easily our first telling result as follows.

Proposition 1. Let $T \in B(\mathscr{H})$ be a hyponormal unilateral weighted shift with Te $e_{n}=\alpha_{n} e_{n+1}$ for all $n \in \mathbb{N}$ and $\gamma=\left\{\gamma_{n}\right\}_{n \in \mathbb{N}}$ the moment sequence (1) associated with $\alpha$, satisfying $\left|\alpha_{n}\right|<$ $\left|\alpha_{n+1}\right|$, for all $n \in \mathbb{N}$. Then, $T$ is near subnormal if and only if the sequence $\left\{\left(\gamma_{n-1} / \gamma_{n}\right)^{1 / 2}\left(\left(\gamma_{n+2} \gamma_{n}-\gamma_{n+1}^{2}\right) /\left(\gamma_{n+1} \gamma_{n-1}-\gamma_{n}^{2}\right)\right)^{1 / 2}\right\}_{n \geq 1}$ is bounded.

The advantage of this result consists of its application to the former sequence (2), for establishing sufficient conditions on the near subnormality.

Theorem 2. Let $\gamma=\left\{\gamma_{n}\right\}_{n \in \mathbb{N}}$ be a positive sequence (2) and $P(z)=\prod_{i=1}^{s}\left(z-\lambda_{i}\right)^{k_{i}}\left(k_{1}+\cdots+k_{s}=r\right)$ its characteristic polynomial with $0<\left|\lambda_{1}\right| \leq\left|\lambda_{2}\right| \leq \cdots<\left|\lambda_{s-1}\right|<\left|\lambda_{s}\right|$. Let $\alpha=\left\{\alpha_{n}\right\}_{n \in \mathbb{N}}$ be the sequence defined by $\alpha_{n}^{2}=\gamma_{n+1} / \gamma_{n}$, satisfying $\left|\alpha_{n}\right|<\left|\alpha_{n+1}\right|$, for all $n \in \mathbb{N}$. Then, the hyponormal operator $T$ associated with unilateral weighted shift $\alpha$ is near subnormal.

Proof. Let $\gamma=\left\{\gamma_{n}\right\}_{n \in \mathbb{N}}$ be a positive sequence (2) and $P(z)=\prod_{i=1}^{s}\left(z-\lambda_{i}\right)^{k_{i}}$ its characteristic polynomial with $0<\lambda_{1} \leq \lambda_{2} \leq \cdots<\lambda_{s-1}<\lambda_{s}$. It follows from the Binet formula that $\gamma_{n}=\sum_{i=1}^{s} \sum_{j=1}^{k_{i}} c_{i, j} n^{j} \lambda_{i}^{n}$, for all $n \in$ $\mathbb{N}$ (see [6]). A straightforward computation leads to have $\lim _{n \rightarrow+\infty}\left\{\left(\gamma_{n-1} / \gamma_{n}\right)^{1 / 2}\left(\left(\gamma_{n+2} \gamma_{n}-\gamma_{n+1}^{2}\right) /\left(\gamma_{n+1} \gamma_{n-1}-\gamma_{n}^{2}\right)\right)^{1 / 2}\right\}=$ $\lambda_{s-1}^{1 / 2}$. By the above Proposition 1, we obtain the desired result.

More generally, suppose that $0<\left|\lambda_{1}\right| \leq\left|\lambda_{2}\right| \leq$ $\cdots \leq\left|\lambda_{s-1}\right| \leq\left|\lambda_{s}\right|$; it may occur in the Binet formula $\gamma_{n}=\sum_{i=0}^{s} p_{i}(n) \lambda_{i}^{n}$, where $p_{i}(n)=\sum_{j=0}^{k_{i}} c_{i, j} n^{j}$, that there exist $\lambda_{s-d}, \ldots, \lambda_{s}(d \geq 1)$ such that $\lambda_{i} \neq \lambda_{j},\left|\lambda_{i}\right|=\left|\lambda_{j}\right|$ for $s-d \leq i \neq j \leq s$ and $\left|\lambda_{j}\right|<\left|\lambda_{s}\right|$ for $1 \leq j \leq s-d-1$. If $\operatorname{deg}\left(p_{s}(z)\right)>\operatorname{deg}\left(p_{j}(z)\right)$, for every $0<j \leq s-1$, then a straightforward computation allows us to establish that $\sup _{n \geq 1}\left\{\alpha_{n}\left(\left(\alpha_{n+1}^{2}-\alpha_{n}^{2}\right) /\left(\alpha_{n}^{2}-\alpha_{n-1}^{2}\right)\right)^{1 / 2}\right\}<+\infty$. Therefore, under the preceding data, the conclusion of Theorem 2 is still valid. Now, let us study the general situation when there exist $k_{1}, \ldots, k_{r}$ with $s-d \leq k_{i} \neq k_{j} \leq s(1 \leq i \neq j \leq r)$ such that $\operatorname{deg}\left(p_{k_{i}}(z)\right)=\operatorname{deg}\left(p_{k_{j}}(z)\right)$; we can also demonstrate that $\sup _{n \geq 1}\left\{\alpha_{n}\left(\left(\alpha_{n+1}^{2}-\alpha_{n}^{2}\right) /\left(\alpha_{n}^{2}-\alpha_{n-1}^{2}\right)\right)^{1 / 2}\right\}<+\infty$. To establish this result, a long and direct computation is necessary; and the following lemma will be useful.

Lemma 3. Let $\gamma=\left\{\gamma_{n}\right\}_{n \in \mathbb{N}}$ be a positive sequence (2) and suppose that its characteristic polynomial is given by $P(z)=$ $\left(z-\lambda_{1}\right)^{s}\left(z-\lambda_{2}\right)^{s}$, where $\lambda_{1} \neq \lambda_{2}$ and $\left|\lambda_{1}\right|=\left|\lambda_{2}\right| \neq 0$. Let $\alpha=\left\{\alpha_{n}\right\}_{n \in \mathbb{N}}$ be the sequence defined by $\alpha_{n}^{2}=\gamma_{n+1} / \gamma_{n}$, satisfying $\left|\alpha_{n}\right|<\left|\alpha_{n+1}\right|$, for all $n \in \mathbb{N}$. Then, we have $\sup _{n \geq 1}\left\{\alpha_{n}\left(\left(\alpha_{n+1}^{2}-\alpha_{n}^{2}\right) /\left(\alpha_{n}^{2}-\alpha_{n-1}^{2}\right)\right)^{1 / 2}\right\}<+\infty$.

The proof of this lemma is more technical and for the reason of clarity we omit it. For the reason of simplicity, we study the general situation, when the Binet formula is given by $\gamma_{n}=p_{s}(n) \lambda_{s}^{n}+p_{s-1}(n) \lambda_{s-1}^{n}+\sum_{i=1}^{s-2} p_{i}(n) \lambda_{i}^{n}$, where $\operatorname{deg}\left(p_{i}(z)\right)<\operatorname{deg}\left(p_{s}(z)\right)=r$ for $1 \leq i \leq s-2, p_{s}(n)=$ $a n^{r}+q_{s}(n)(a \neq 0)$, and $p_{s-1}(n)=b n^{r}+q_{s-1}(n)(b \neq 0)$ with $q_{s}(n)=\sum_{j=1}^{r-1} a_{j} n^{j}$ and $q_{s-1}(n)=\sum_{j=1}^{r-1} b_{j} n^{j}$. Since $\lim _{n \rightarrow+\infty} p_{i}(n) \lambda_{i}^{n} / p_{s}(n) \lambda_{s}^{n}=0$, for every $1 \leq i \leq s-2$, Lemma 3 implies the following.

Lemma 4. Let $\gamma=\left\{\gamma_{n}\right\}_{n \in \mathbb{N}}$ be a positive sequence (2) and suppose that its characteristic polynomial is $P(z)=$ $\left(z-\lambda_{s}\right)^{r}\left(z-\lambda_{s-1}\right)^{r} \prod_{j=2}^{s-3}\left(z-\lambda_{j}\right)^{m_{j}}$, where $\lambda_{s} \neq \lambda_{s-1},\left|\lambda_{s}\right|=$ $\left|\lambda_{s-1}\right| \neq 0$ and $\left|\lambda_{j}\right|<\left|\lambda_{s}\right|$, for all $1 \leq j \leq s-2$. Let $\alpha=\left\{\alpha_{n}\right\}_{n \in \mathbb{N}}$ be the sequence defined by $\alpha_{n}^{2}=\gamma_{n+1} / \gamma_{n}$, satisfying $\left|\alpha_{n}\right|<\left|\alpha_{n+1}\right|$ for all $n \in \mathbb{N}$. Then, we have $\sup _{n \geq 1}\left\{\alpha_{n}\left(\left(\alpha_{n+1}^{2}-\alpha_{n}^{2}\right) /\left(\alpha_{n}^{2}-\alpha_{n-1}^{2}\right)\right)^{1 / 2}\right\}<+\infty$.

Lemmas 3 and 4 permit us to formulate the following extension of Theorem 2 .

Theorem 5. Let $\gamma=\left\{\gamma_{n}\right\}_{n \in \mathbb{N}}$ be a positive sequence (2) and $P(z)=\prod_{i=1}^{s}\left(z-\lambda_{i}\right)^{k_{i}}$ its characteristic polynomial with $0<$ $\left|\lambda_{1}\right| \leq\left|\lambda_{2}\right| \leq \cdots \leq\left|\lambda_{s-1}\right| \leq\left|\lambda_{s}\right|$. Let $\alpha=\left\{\alpha_{n}\right\}_{n \in \mathbb{N}}$ be the sequence defined by $\alpha_{n}^{2}=\gamma_{n+1} / \gamma_{n}$, such that $\left|\alpha_{n}\right|<\left|\alpha_{n+1}\right|$ for all $n \in \mathbb{N}$. Then, the hyponormal operator $T$ associated with unilateral weighted shift $\alpha$ is near subnormal.

We manage to reassemble the above results as follows.

Theorem 6. Let $T \in B(H)$ be a hyponormal operator such that $T e_{n}=\alpha_{n} e_{n+1}(n \geq 0)$. Suppose that the sequence of moment $\gamma=\left\{\gamma_{n}\right\}_{n \in \mathbb{N}}$ is a positive sequence (2). Let $\alpha=\left\{\alpha_{n}\right\}_{n \in \mathbb{N}}$ be the sequence defined by $\alpha_{n}^{2}=\gamma_{n+1} / \gamma_{n}$, satisfying $\left|\alpha_{n}\right|<\left|\alpha_{n+1}\right|$, for all $n \in \mathbb{N}$. Then, $T$ is near subnormal. 
In [8], an important class of subnormal weighted shifts is explored by considering measures $\mu$ with two atoms $\lambda_{1}$ and $\lambda_{2}$. A sequence $\left\{\gamma_{n}\right\}_{n \geq 0}$ such that $\gamma_{0}=1, \gamma_{1}$ and $\gamma_{n+1}=a_{0} \gamma_{n}+a_{1} \gamma_{n-1}$ (for $n \geq 1$ ) is a moment sequence if and only if $P\left(\gamma_{1}\right) \leq 0$. As a matter of fact, we study the fallout of our approach by providing a connection between hyponormality, near subnormality and subnormality for this class of operators of weighted shifts.

Proposition 7. Let $\gamma=\left\{\gamma_{n}\right\}_{n \in \mathbb{N}}$ be a positive sequence (2) and $P(z)=\left(z-\lambda_{1}\right)\left(z-\lambda_{2}\right)$ its characteristic polynomial with $0<$ $\lambda_{1} \leq \lambda_{2}$. Let $\alpha=\left\{\alpha_{n}\right\}_{n \in \mathbb{N}}$ be the sequence defined by $\alpha_{n}^{2}=$ $\gamma_{n+1} / \gamma_{n}$ and $T$ the operator associated with unilateral weighted shift $\alpha$. Suppose that $\alpha_{n} \neq \alpha_{n+1}$, for all $n \in \mathbb{N}$. Then, the following five statements are equivalent: (i) $\alpha_{1}>\alpha_{0}$; (ii) T is hyponormal; (iii) $T$ is $k$-hyponormal for all $k \in \mathbb{N}$; (iv) $T$ is near subnormal; (v) $T$ is subnormal.

Similar to the subnormal completion problem (see [1, $2])$, the NSCP can be formulated as follows: "Let $\alpha^{(m)}=$ $\left\{\alpha_{n}\right\}_{0}^{m}$ be a finite collection of positive numbers, find necessary and sufficient conditions on $\alpha_{m}$ to guarantee the existence of a near subnormal weighted shift whose initial weights are given by $\alpha^{(m)}$ ". The first obstructions encountered for solving this problem are the natural necessary condition for the existence of the hyponormal completion. That is, once we know that $\alpha^{(m)}: \alpha_{0}, \ldots, \alpha_{2 m}(m \geq 1)$ admits a $k$-hyponormal completion which is recursively generated, the condition $\alpha_{n}<$ $\alpha_{n+1}$ is not easy to be satisfied in order to apply Theorem 6 . When $m \geq 2$, the problem becomes highly nontrivial. For $m=1$, the strategy to solve NSCP is as follows. Given $\alpha^{(1)}: \alpha_{0}, \alpha_{1}, \alpha_{2}$ such that $\alpha_{0}<\alpha_{1}<\alpha_{2}$, set $\gamma_{0}=1, y_{1}=$ $\gamma_{0}^{2}, y_{2}=\alpha_{0}^{2} \alpha_{1}^{2}$, and $\gamma_{3}=\alpha_{0}^{2} \alpha_{1}^{2} \alpha_{2}^{2}$. First, we use these terms to construct a recursive sequence $\left\{\gamma_{n}\right\}_{n \geq 0}$ of order 2 , by setting $\gamma_{n}=\rho_{0} \gamma_{n-1}+\rho_{1} \gamma_{n-2}$ for all $n \geq 2$. A straightforward calculation gives $\rho_{0}=\alpha_{1}^{2}\left(\alpha_{2}^{2}-\alpha_{0}^{2}\right) /\left(\alpha_{1}^{2}-\alpha_{0}^{2}\right), \rho_{1}=\alpha_{1}^{2} \alpha_{2}^{2}\left(\alpha_{1}^{2}-\right.$ $\left.\alpha_{2}^{2}\right) /\left(\alpha_{1}^{2}-\alpha_{0}^{2}\right)$ and $\alpha_{n+2}^{2}=\alpha_{n+1}^{2}+\left(\alpha_{n-1}^{2}\left(\alpha_{n+1}^{2}-\alpha_{n}^{2}\right) / \alpha_{n+1}^{2}\left(\alpha_{n}^{2}-\right.\right.$ $\left.\left.\alpha_{n-1}^{2}\right)\right), n \geq 1$, where $\alpha_{n}^{2}=y_{n+1} / y_{n}$. Thus, we obtain $\alpha_{n}>\alpha_{n-1}$, for every $n \geq 0$. And the completion $\widehat{\alpha}=\{\alpha\}_{n \geq 0}$ of $\alpha^{(2)}$ is hyponormal and recursively generated, and the condition $\alpha_{n}<\alpha_{n-1}$ is satisfied. It follows from Theorem 6 that $W_{\alpha}$ is near subnormal. As a matter of fact, we mange to have the following result.

Proposition 8. Let $\alpha$ : $\alpha_{0}<\alpha_{1}<\alpha_{2}$ be an initial segment of positive weights. Then, $\alpha$ has a near subnormal completion.

\section{The Moment of Distributions and the Near Subnormality of Unilateral Weighted Shifts}

In this section, we are interested in formulating some results in the moment of distributions of discrete support, with a view to characterize the near subnormality and its closed relation with the subnormality of weighted shifts. Let $H$ be a separable Hilbert space and $\left\{e_{n}\right\}_{n \geq 0}$ its orthonormal basis. Let $\alpha=\left\{\alpha_{n}\right\}_{n \geq 0}$ be a bounded sequence of nonnegative real numbers and $W_{\alpha}$ the bounded operator defined by $W_{\alpha} e_{n}=\alpha_{n} e_{n+1}$.
Let $\gamma=\left\{\gamma_{n}\right\}_{n \geq 0}$ be the sequence of moments associated with $W_{\alpha}$. By Berger's theorem, $W_{\alpha}$ is a subnormal operator if and only if there exists a nonnegative Borelean measure $\mu$, which is called a representing measure of $\gamma=\left\{\gamma_{n}\right\}_{n \geq 0}$, with $\operatorname{supp}(\mu) \subset\left[0,\left\|W_{\alpha}\right\|^{2}\right]$ such that $\gamma_{n}=\int_{0}^{\left\|W_{\alpha}\right\|^{2}} t^{n} d \mu(t), \quad(n \geq$ $0)$, where $\left\|W_{\alpha}\right\|^{2}=\sup _{n \geq 0} \alpha_{n}$. Hence, the moment problem and subnormal weighted shifts are closely related. And it was shown in [8] that a sequence (2) $\gamma=\left\{\gamma_{n}\right\}_{n \geq 0}$ admits a generating measure (not necessary positive) if and only if its characteristic (minimal) polynomial $P_{\gamma}$ has distinct roots, with $\operatorname{supp}(\mu)=Z\left(P_{\gamma}\right)$, the set of zeros of $P_{\gamma}$ (for more details, see Proposition 2.4 of [8]). It was pointed out in [9] that if $P_{\gamma}(z)=\prod_{i=0}^{s}\left(z-\lambda_{i}\right)^{p_{i}}$, with $p_{i} \geq 2$ for some $i$, then $\gamma$ is a moment sequence for some distribution $T=\sum_{i=0}^{p} \sum_{j=1}^{p_{i}} \rho_{i, j} \delta_{\lambda_{i}}^{(j)}$, where $\delta_{\lambda_{i}}^{(j)}$ is the $j$ th derivative in the meaning of distribution of the Dirac measure $\delta_{\lambda_{i}}$ (see $[10]$ ). To find this distribution (supported by a compact $K$ ) interpolating $\gamma$, the Binet formula plays a primordial role. Indeed, let $K$ be compact subset of $\mathbb{R}$ (or $\mathbb{C}$ ), $U$ a neighborhood of $K$, and consider the function $g_{K}$ of class $C^{\infty}$ satisfying the following three conditions: (i) $g_{K}(t)=1$ for every $t \in K$; (ii) $0 \leq g_{K}(t) \leq 1$ for every $t \in \mathbb{R}$ or $\mathbb{C}$; and (iii) $g_{K}(t)=0$ for every $t \in \mathbb{R}-U$ (or $\mathbb{C}-U$ ). It was established in [10] that for a distribution $T$ of compact support $K$, the real (or complex) number $\gamma_{n}=\left\langle T \mid g_{K}(t) t^{n}\right\rangle$ is independent of the function $g_{K}(t)$, for every $n \in \mathbb{N}$ (see Lemma 1 of [10]). The number $\gamma_{n}=\left\langle T \mid g_{K}(t) t^{n}\right\rangle=\left\langle T \mid t^{n}\right\rangle(n \geq 0)$ is called the moment (or power moment) of order $n$ of the distribution $T$.

Let $\delta_{i}$ be the Dirac measure at the point $\lambda_{i}$ and $\delta_{i}^{(j)}$ its $j$ th derivative. It is well known that $\delta_{i}$ and $\delta_{i}^{(j)}$ define two distributions on the space of polynomial functions on $\mathbb{R}$ (or $\mathbb{C}$ ). Moreover, every distribution of discrete support $\left\{\lambda_{1}, \ldots, \lambda_{s}\right\}$ can be written under the form $T=\sum_{i=0}^{p} \sum_{j=1}^{p_{i}} c_{i, j} \delta_{\lambda_{i}}^{(j)}$ (see, [11] e.g.). We denote by $\mathbf{D}_{K}$ the $\mathbb{C}$-vector space of distributions of discrete support, contained in $K$. Hence, the preceding discussion and Theorem 6, allow us to derive the following result.

Theorem 9. Let $W \in B(H)$ be a hyponormal operator such that $W e_{n}=\alpha_{n} e_{n+1}(n \geq 0)$ and $\left|\alpha_{n}\right|<\left|\alpha_{n+1}\right|$, for all $n \in \mathbb{N}$. Suppose that the sequence of moments $\gamma=\left\{\gamma_{n}\right\}_{n \in \mathbb{N}}$ is a positive sequence and there exists a distribution $T \in \mathbf{D}_{K}$ such that $\gamma_{n}=$ $\left\langle T \mid t^{n}\right\rangle$, for every $n \geq 0$. Then, $W$ is near subnormal.

The natural extension of the $K$-moment problem can be formulated as follows. Let $K$ be a closed subset of $\mathbb{R}$ and $\left\{\gamma_{n}\right\}_{n \geq 0}$ a sequence of $\mathbb{C}$. The associated distributional $K$-moment problem consists of finding a distribution $T$ of support contained in $K$ such that

$$
\gamma_{n}=\left\langle T \mid t^{n}\right\rangle, \quad \text { for every } n(n \geq 0) .
$$

A distribution $\mu$ solution of problem (3) is called a representing distribution of the sequence $\gamma=\left\{\gamma_{n}\right\}_{n \geq 0}$. Therefore, the equivalent form of Theorem 9 can be expressed as follows.

Proposition 10. Let $W \in B(H)$ be a hyponormal operator such that $W e_{n}=\alpha_{n} e_{n+1}(n \geq 0)$ with $\left|\alpha_{n}\right|<\left|\alpha_{n+1}\right|$, for all 
$n \in \mathbb{N}$. Suppose that the sequence of moments $\gamma=\left\{\gamma_{n}\right\}_{n \in \mathbb{N}}$ is a positive sequence. If the distributional moment problem (3) owns a solution $\mu \in \mathbf{D}_{K}$, then $W$ is near subnormal.

In light of Riesz theorem, it is well known that if $T$ is a positive distribution then $\langle T \mid \varphi\rangle=\int_{K} t^{n} d \mu(t)$, where $\mu$ is a measure, and we write $T=T_{\mu}=\mu$ (see, [11] e.g.).). As a consequence, we have the following result.

Theorem 11. Let $W \in B(H)$ be a hyponormal operator given by $W e_{n}=\alpha_{n} e_{n+1}(n \geq 0)$ such that its sequence of moments $\gamma=\left\{\gamma_{n}\right\}_{n \in \mathbb{N}}$ is a positive sequence. Let $\alpha=\left\{\alpha_{n}\right\}_{n \in \mathbb{N}}$ be the sequence defined by $\alpha_{n}^{2}=\gamma_{n+1} / \gamma_{n}$, satisfying $\left|\alpha_{n}\right|<\left|\alpha_{n+1}\right|$, for all $n \in \mathbb{N}$. If the moment problem (3) owns a positive representing distribution $\mu \in \mathbf{D}_{K}$, then $W$ is subnormal.

\section{Acknowledgments}

The authors would like to thank the anonymous referee for his (or her) useful remarks and suggestions that improved this paper. M. Rachidi is an Associate with "Group of DEFA."

\section{References}

[1] R. E. Curto and L. A. Fialkow, "Recursively generated weighted shifts and the subnormal completion problem," Integral Equations and Operator Theory, vol. 17, no. 2, pp. 202-246, 1993.

[2] R. E. Curto and L. A. Fialkow, "Recursively generated weighted shifts and the subnormal completion problem. II," Integral Equations and Operator Theory, vol. 18, no. 4, pp. 369-426, 1994.

[3] P. R. Halmos, A Hilbert Space Problem Book, Van Nostrand, Princeton, NJ, USA, 1967.

[4] W. Gong-Bao and M. Ji-Pu, "Near subnormality of weighted shifts and the answer to the Hilbert space problem 160," Northeastern Mathematical Journal, vol. 17, no. 1, pp. 45-48, 2001.

[5] W. Gong-Bao and M. Ji-Pu, "Near subnormal operators and subnormal operators," Science in China A, vol. 33, no. 1, pp. 1-9, 1990.

[6] F. Dubeau, W. Motta, M. Rachidi, and O. Saeki, "On weighted $r$ generalized Fibonacci sequences," The Fibonacci Quarterly, vol. 35, no. 2, pp. 102-110, 1997.

[7] L. A. Fialkow, Positivity, Extensions and the Truncated Moment Problem, Multi-Variable Operator Theory, vol. 185 of Contemporary Mathematics, American Mathematical Society, Providence, RI, USA, 1995.

[8] R. Ben Taher, M. Rachidi, and E. H. Zerouali, "Recursive subnormal completion and the truncated moment problem," The Bulletin of the London Mathematical Society, vol. 33, no. 4, pp. 425-432, 2001.

[9] C. E. Chidume, M. Rachidi, and E. H. Zerouali, "Solving the general truncated moment problem by the $r$-generalized Fibonacci sequences method," Journal of Mathematical Analysis and Applications, vol. 256, no. 2, pp. 625-635, 2001.

[10] B. Bernoussi, M. Rachidi, and O. Saeki, "Factorial Binet formula and distributional moment formulation of generalized Fibonacci sequences," The Fibonacci Quarterly, vol. 42, no. 4, pp. 320-329, 2004.

[11] L. Schwartz, Théorie des Distributions, Hermann, 1966. 


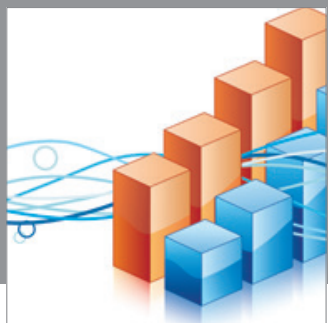

Advances in

Operations Research

mansans

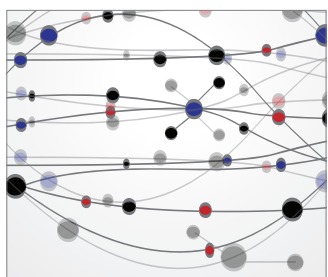

The Scientific World Journal
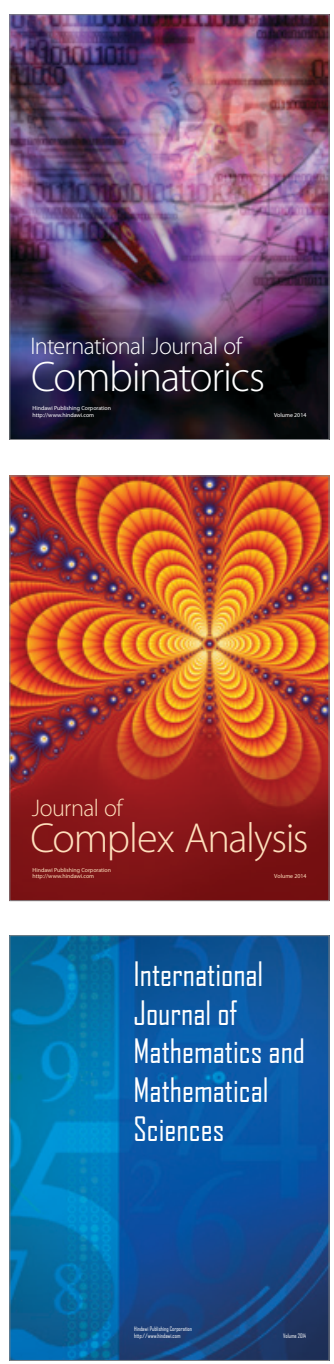
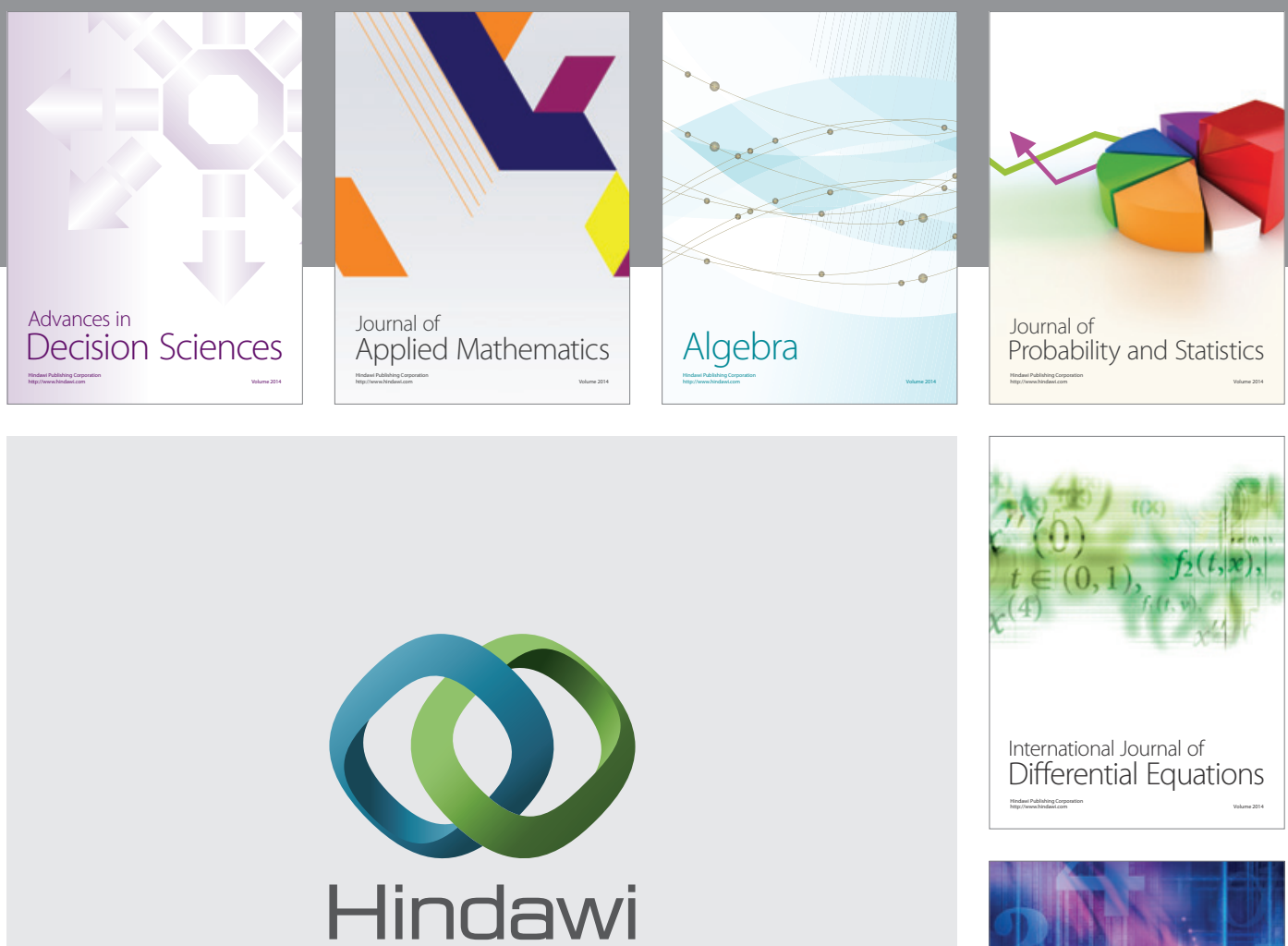

Submit your manuscripts at http://www.hindawi.com
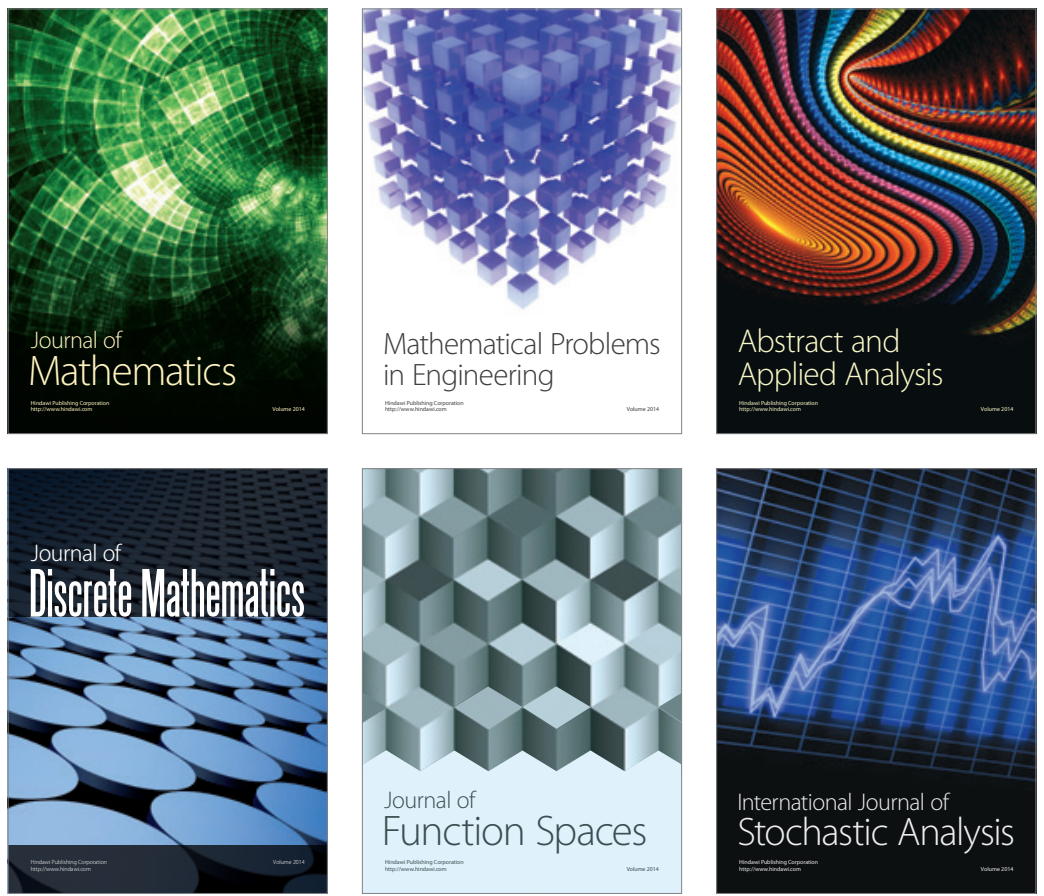

Journal of

Function Spaces

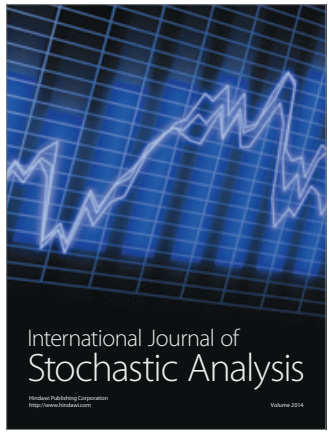

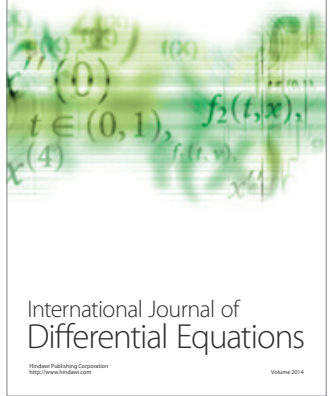
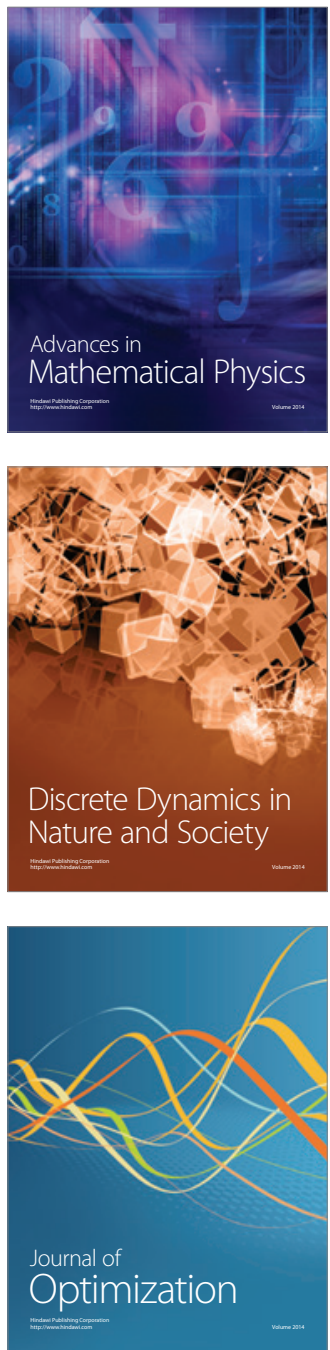